(c) The Author(s), 2021. Published by Cambridge University Press on behalf of The Nutrition Society. This is an Open Access article, distributed under the terms of the Creative Commons Attribution licence (http://creativecommons.org/licenses/by/4.0/), which permits unrestricted re-use, distribution, and reproduction in any medium, provided the original work is properly cited.

\title{
Could consumption of yam (Dioscorea) or its extract be beneficial in controlling glycaemia: a systematic review
}

\author{
Waad Z. Alharazi, Anthony McGowen, Peter Rose and Preeti H. Jethwa* \\ Division of Food, Nutrition and Dietetics, School of Biosciences, University of Nottingham, Sutton Bonington Campus, \\ Loughborough LE12 5RD, UK \\ (Submitted 1 July 2021 - Final revision received 2 September 2021 - Accepted 9 September 2021 - First published online 15 September 2021)
}

\section{Abstract}

Yam (Dioscorea spp.) and its associated extracts have been shown to possess a variety of biological activities and identified as beneficial in the control of glycaemia in patients with type II diabetes mellitus (T2DM). The objective was to conduct a systematic search of the literature to investigate whether yam and its extract can improve glycaemia and whether the consumption of yam could be beneficial for managing T2DM. Using the Preferred Reporting Items for Systematic Reviews and Meta-Analyses guidelines and the Population, Invention, Comparison and Outcome framework, three databases (PubMed, Scopus and Web of Science) were searched using a key term strategy. Strict inclusion criteria were employed to identify all relevant and available studies. The quality of these studies was assessed using SYRCLE's Risk of Bias tool. Ten studies were included, and all studies consisted of findings from rodent models of diabetes, including animals consuming high fat diets or genetic models of diabetes. All ten studies showed that the consumption of yam and/or its extracts (containing dioscin, dioscorin, diosgenin, DA-9801/02 or Chinese yam polysaccharides) improved glycaemia. These included improvements in fasting blood glucose and reductions in glucose and increase in insulin levels following a glucose tolerance test. Furthermore, significant changes in body weight and adiposity were observed in nine studies, these included improvements in lipid biomarkers in four and reductions in inflammatory markers in one. The current work indicates that the consumption of yam or its extracts can be beneficial for improving blood glucose; however, the molecular mechanism for these effects remains largely unknown. Future trials on human subjects are warranted.

Key words: Yam: Dioscorea: Type II diabetes: High fat diet: Glycaemia: Rodent

Diabetes mellitus is a non-communicable metabolic disease projected to affect 366 million by $2030^{(1-3)}$. The most common form of diabetes is type II diabetes mellitus (T2DM) which often begins with obesity associated with insulin resistance and glucose tolerance leading to hyperglycaemia, impaired $\beta$-cell function and a decrease in insulin secretion ${ }^{(4)}$. Furthermore, impairment in lipid and lipoprotein metabolism, increase in oxidative stress, diminished vascular endothelial function and high blood pressure are also common in T2DM ${ }^{(5,6)}$. Chronic exposure to these complications often leads to health conditions including peripheral neuropathy, retinopathy and nephropathy alongside an increased mortality rate ${ }^{(7)}$.

Therefore, controlling blood glucose is important to prevent diabetic complications and to improve health of patients. While a number of hypoglycaemic agents have been developed, based on current understanding of the pathophysiology of T2DM, their use results in a myriad of side effects and the initial improvements in glycaemia are usually not sustained ${ }^{(8,9)}$.

Obesity is a risk factor for the development of T2DM, and dietary management is thought to reduce the burden on islet cells and thus improve glucose levels, inflammation and lipid profile ${ }^{(10)}$. Recent evidence suggests that the regular consumption of foods with bioactive compounds may benefit health related to prevention or management of chronic diseases ${ }^{(11-13)}$.

Yam (Dioscorea), an angiosperm (flowering plant) not botanically related to sweet potato (Ipomoea), is commonly consumed in the Asian and African continents ${ }^{(14)}$. In the African populations, the prevalence of T2DM ranges from $3.5 \%$ in rural area to $7.5 \%$ in urban area ${ }^{(15,16)}$. In Asia, yam has been used in traditional Chinese medicine as a natural medicine for $\mathrm{T}_{2} \mathrm{DM}^{(17)}$.

Of particular interest in this region are the numerous extracts, which include allantoin, dioscorin, sapogenins, prosapogenin,

Abbreviations: DBP, diastolic blood pressure; FBG, fasting blood glucose; GTT, glucose tolerance test; HFD, high fat diet; HOMA-IR, homoeostatic model assessment of insulin resistance; ITT, insulin tolerance test; MCR, metabolic clearance rate; MMP, matrix metalloproteinase; SBP, systolic blood pressure; STZ, streptozotocin; TC, total cholesterol; T2DM, type II diabetes mellitus.

* Corresponding author: Preeti H. Jethwa, email preeti.jethwa@nottingham.ac.uk 
gracillin, choline, L-arginine, polysaccharides and proteins. Several in vitro and in vivo studies have highlighted the antidiabetic action of a number of these extracts, including dioscorea ethanol extract ${ }^{(18)}$, total saponins ${ }^{(19-22)}$ allantoin ${ }^{(23,24)}$, water soluble polysaccharides ${ }^{(25)}$, DA-9801(26) and diosgenin ${ }^{(27,28)}$. However, many of these studies have been conducted in animal models in which diabetes was induced by streptozotocin ${\text { (STZ })^{(19-22)} \text { or alloxan }}^{(18,25)}$. These are popular methods but induce hyperglycaemia via the destruction of the pancreatic islets and do not mimic the insulin resistance presented in human patients with $\mathrm{T}_{2} \mathrm{DM}^{(29)}$.

Therefore, we conducted a systematic review to search the literature to investigate whether yam and its extract can improve glycaemia in diet-induced and spontaneous T2DM in vivo models and determine whether the consumption of these could be a diet modification.

\section{Method}

The review was constructed in accordance with the Preferred Reporting Items for Systematic Reviews and Meta-Analyses guidelines $^{(30)}$.

\section{Searching strategy}

A computerised search of the literature was performed using three databases (PubMed, Scopus and Web of Science) between April 2020 and June 2020. The searching process followed the Population, Invention, Comparison and Outcome framework. The population was T2DM animal models or human patients diagnosed with T2DM, the intervention was yam or yam extracts in comparison with controls who do not receive the intervention and measuring the outcome is the effect of the yam intervention on complications associated with T2DM such as insulin sensitivity and glucose tolerance. Search of terms was conducted through the literature to define the keywords: yam OR 'yam extract' OR Dioscorea AND diabetes OR antidiabet $\times O R$ glycaem $\times$ OR insulin OR glucose OR T2DM. Two independent reviewers (WA and AM) assessed the titles, abstracts and full articles, based on strict inclusion and exclusion criteria; any disagreements with the section of the article were resolved through discussion. Full articles of the selected titles were retrieved, and the reference lists of these were searched to identify any additional publications.

Selected studies criteria. All related articles from inception were considered as there have been no previous systematic reviews conducted to investigate the relationship of yam and its phytochemicals to the anti-diabetic effects identified during our search.

\section{Inclusion criteria.}

- Only articles written in English were eligible to avoid any misleading translations.

- All studies must have described either animal models with diet induced T2DM or human participants who have been diagnosed with T2DM by a medical profession.

- Any yam and yam extracts were considered.
- All studies must have a measure of glycaemic parameters.

- Randomised clinical trials.

- Fully published studies.

\section{Exclusion criteria.}

- Chemically induced hyperglycaemia using pharmaceutical agents (e.g. STZ).

- Non-diabetic model.

- In vitro cell studies exploring the cellular mechanisms.

- Systematic reviews or critical reviews.

- Traditional Chinese medicine or any traditional medicine that contains other plants in addition to or alternative to yam.

\section{Measured outcomes}

The primary outcomes of this review are the effect on glycaemic parameters such as fasting blood glucose (FBG), $\mathrm{HbA}_{1} \mathrm{c}$, glucose tolerance test (GTT), insulin levels, homoeostatic model assessment of insulin resistance (HOMA-IR), insulin-glucose ratio, insulin sensitivity index, insulin tolerance test (ITT), metabolic clearance rate (MCR) and adiposity insulin resistance index, while secondary measurements were factors associated with glycaemic control. These include body weight, lipid profile (total fat, white adipose tissue, total cholesterol (TC), TAG, LDL and HDL and NEFA), blood pressure (systolic blood pressure (SBP) and diastolic blood pressure (DBP)) and inflammatory parameters (leptin, IL-1 $\beta$, IL-10, matrix metalloproteinase (MMP), NF-кB).

\section{Data extraction}

A standard data extraction form was used to obtain data from the studies and charted using Excel (Microsoft Excel). Data extracted included title, author, publication year, country, study population, sample size, diabetic model, exposure to yam genus or yam extracts and outcomes $\left(\mathrm{FBG}, \mathrm{HbA}_{1} \mathrm{c}\right.$, glucose levels following GTT, insulin levels, HOMA-IR, MCR and adiposity insulin resistance index), body weight change, lipid profiles (total fat, white adipose tissue, TC, TAG, LDL and HDL and NEFA), blood pressure (SBP and DBP) and inflammatory parameters (leptin, IL-1 $\beta$, IL-10, MMP, NF-אB)). The results from each study alongside statistical outcomes were also extracted.

\section{Data analysis}

The relevant results were expressed in tables. The key characteristics of the selected papers included the study design, population, model used, number of the sample, outcome measures and doses of intervention groups. The significant effects in response to the intervention were charted to compare across the article retrieved. Raw values for the primary outcome measures were not reported consistently across all studies; therefore, we were unable to compare the magnitude of the effect on the primary outcome measures and conduct a meta-analysis.

\section{Quality assessment}

The SYRCLE's Risk of Bias tool was used to assess quality assessment due to the lack of human participant studies. This is an adapted version of the Cochrane Risk of Bias tool ${ }^{(31)}$ consisting of ten items relating to six types of bias. Items 1, 3, 8, 9 and 10 are 
adopted from the Cochrane Risk of Bias tool, while items 2, 4, 5,6 and 7 have been adapted or replaced to allow for appropriate assessment of animal studies ${ }^{(32)}$ (online Supplementary Table S1). Signalling criteria were used to determine and assign a judgement of low, high or unclear risk of bias.

The quality assessment examined multiple types of bias: selection, performance and direction, attrition and reporting Selection bias (items 1, 2 and3) was assessed by sequence generation, baseline characteristics and allocation concealment. Performance bias (items 4 and 5) was assessed by randomised housing and blinding relating to researchers and/or animal caregivers. Detection bias (items 6 and 7) assessed any random outcome assessment and blinding as it can lead to multiple types of bias. Attrition bias (item 8) was explored by assessing incomplete outcome data, while reporting bias (item 9) assessed selective outcome reporting. Other sources of bias were covered by item 10 (online Supplementary Table S1).

\section{Results}

Eligibility of studies

Using three electronic databases (PubMed/Medline, Scopus and WoS), we identified 967 papers published between 1962 and
April 2020. Following the removal of duplicates and an initial title screen, 215 studies were assessed in more detail. Of these, fiftyeight were evaluated against stringent inclusion/exclusion criteria, thirty-four used medically induced diabetic models, seven utilised a mixture of compounds which contained extracts from sources other than yam, seven did not measure glucose levels and four did not include yam. This left ten studies eligible for inclusion (Fig. 1).

\section{Quality assessment}

All ten papers had 'Fair' as a final judgement for quality (Table 1 and online Supplementary S1). Based on the assessed bias criteria, the only study that scored 'High Risk' at the 'Selection Bias' questions 1, 2, 3 was ref. [33]. While 'Performance and Detection bias' questions 4 and 6 had unclear and low risk in all studies, questions 5 and 7 which assess the blinding for caregiver and investigators were judged as 'High Risk'; however, this may not affect the overall judgement where ref. [34] found in their meta-analysis that blinding in animal trials is not statistically significant. In regard to 'Attrition Bias', question 8 highlighted two papers as 'High Risk' which are ref. [35] where eight out of ten mice outcomes data were reported and ref. [36] where eight out of fourteen mice outcomes data were reported.

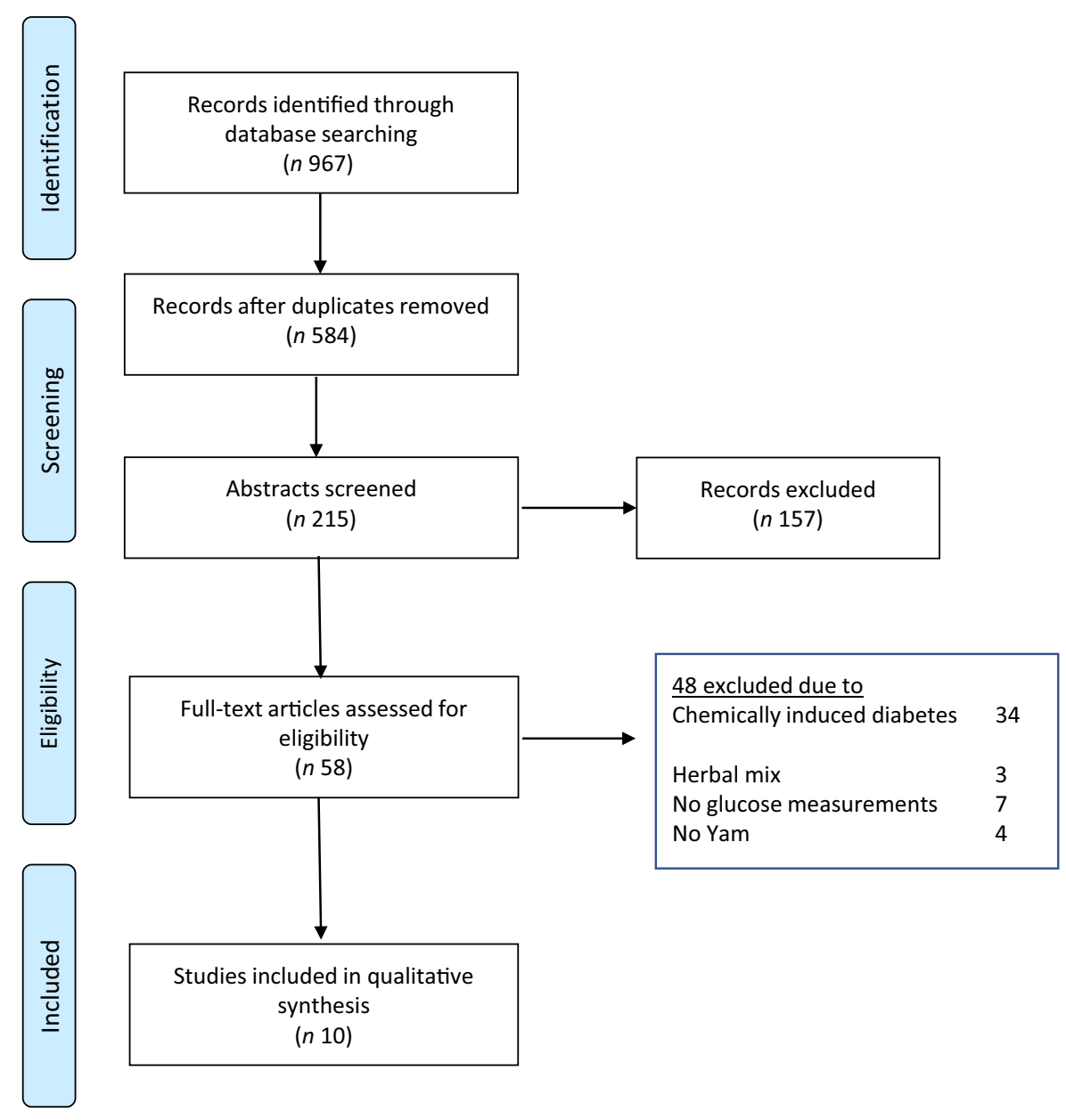

Fig. 1. Flow diagram demonstrating the identification and selection of relevant research (PRIMSA, 2015). 
Table 1. SYCRLE tool for Risk of Bias (RoB) of selected studies

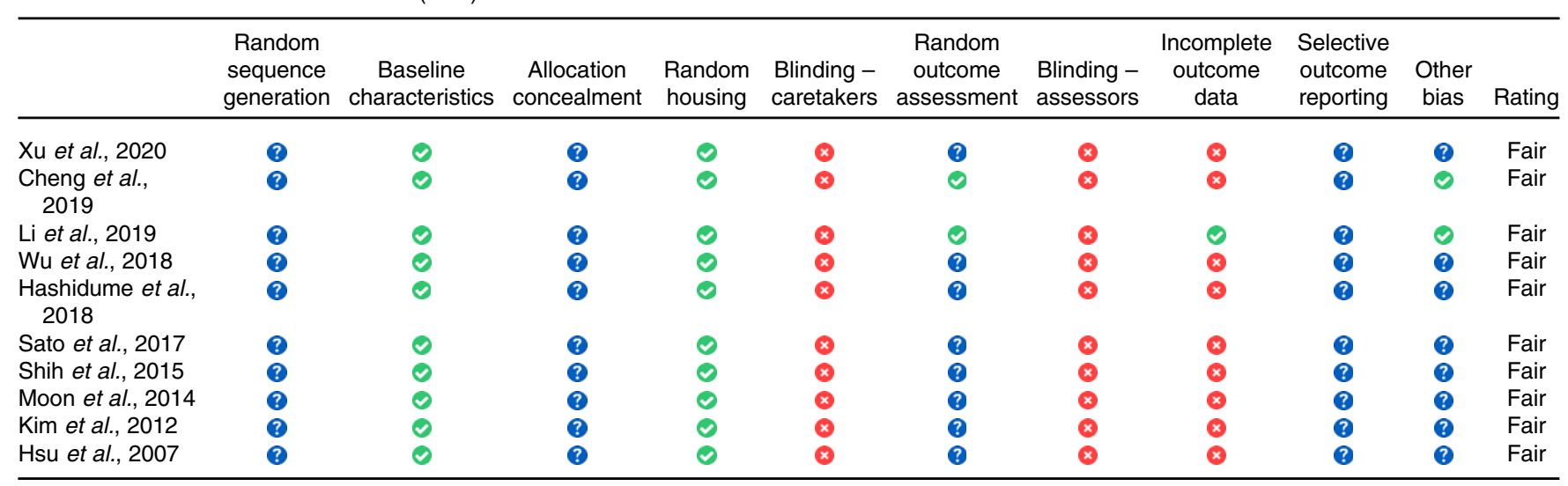

?. unclear risk of bias, $\bullet$, low risk of bias, $\mathbb{\otimes}$, high risk of bias, according to SYCRLE recommendations.

\section{Study characteristics}

The characteristics of the studies are summarised in Table 2. The selected studies were located in Asian countries, though no restraints were placed on location. The experimental length varied from 4 weeks to 19 weeks with an average of 11 weeks. No studies included human participant diagnosed with T2DM, and all studies were carried out in rodent model, although two studies utilised in vitro models as part of their study design ${ }^{(33,35)}$. In the rodent model, T2DM was induced by consuming high fat diet (HFD) in rats or mice, the KK-A ${ }^{\mathrm{y}}$ mice, which spontaneously exhibit T2DM and the $d b / d b$ mice, a genetic model of T2DM, were fed HFD, while the OLETF mice fed normal diet; one study, ref. [37] fed the rodent model rats with high fructose diet to induce hyperglycaemia. The studies utilised a variety of Dioscorea species although two studies did not mention the yam species ${ }^{(38,39)}$. A variety of yam extracts were used in nine of the studies; these included components, dioscin ${ }^{(35,38)}$, dioscorin ${ }^{(39,40)}$, diosgenin ${ }^{(41)}$, DA-9801 and DA-9802 ${ }^{(33,42)}$ and Dioscorea esculenta powder ${ }^{(43)}$, while one study used raw material of Dioscorea Opposita (a synonym of two species of yam Dioscorea polystachya and Dioscorea oppositifolia) ${ }^{(37)}$. The yam and/or its extracts were obtained via various methods; five articles reported their extraction methods from raw yam while two articles purchased the yam from external sources $^{(33,35-37,40-42)}$. The extraction methods included aqueous ethanol extraction from dried yam ${ }^{(33,35,42)}$, water extraction and alcohol precipitation method and raw flush sample mixed with Tris buffer and purified with DE-52 ion exchange chromatography ${ }^{(36,40)}$. These were administered either orally or with saline through gavage at varying doses ranging from 5 to $100 \mathrm{mg} / \mathrm{kg}$. Biochemical measurements included measurements of glycaemia (FBG, GTT, HbA1c, HOMA-IR, insulin-glucose ratio, insulin sensitivity index, ITT, MCR and adiposity insulin resistance index), lipid profile (total fat, white adipose tissue, TC, TAG, LDL and HDL and NEFA), blood pressure (SBP and DBP) and inflammatory markers (leptin, IL-1 $\beta$, IL-10, MMP, NF-кB; Tables 3 and 4).

\section{Effects of yam extract on measurements of glycaemia}

Fasting blood glucose/g/ucose tolerance test. All the included studies measured glucose and, although these were measured via a variety of methods (FBG/GTT), they all showed that treatment with either yam or its extract led to significant improvements in glucose tolerance compared with the controls (Table 2). FBG was reported in all studies except ref. [39,40], while GTT was measured in five of the ten studies ${ }^{(35,37,39,40,42)}$. Dioscin, dioscorin, DA-9801, DA-9802, diosgenin and CYP improved FBG $(P<0.05)$, while dioscin, dioscorin and DA-9802 were shown to improve GTT from 30 to 120 min after oral or intraperitoneal glucose load $(P<0.05)$. The lowest working doses of yam or yam extract ranged from 10 to $100 \mathrm{mg} / \mathrm{kg}$ in the HFD model, while in the genetic models of diabetes (KK- $\mathrm{A}^{\mathrm{y}}, d b / d b$, OLEFT) the lowest working doses ranged from 0.5 to $30 \mathrm{mg} / \mathrm{kg}$ (Table 3).

HbA1c. HbA1c was measured in two of the ten studies; both showed that the consumption of yam or its extract significantly reduced $\mathrm{HbA1}^{(35,37)}$. Xu et al. showed that dioscin reduced $\mathrm{HbA1c}$ in $\mathrm{KK}-\mathrm{A}^{\mathrm{y}}$ at all doses, including the lowest dose of 20 $\mathrm{mg} / \mathrm{kg}$, although this dose had no effect on FBG, while Hsu et al. showed that the consumption of D. Opposita Thunb had a significant reduction $(P<0.05)$ (Table 3$)$

Insulin. Fasting insulin levels were measured in three of the ten papers and all showed significant decrease in insulin in a dosedependent manner $(P<0 \cdot 05 \text {; Table } 3)^{(35,38,42)}$. HFD mice treated with dioscin (10 and $20 \mathrm{mg} / \mathrm{kg}$ ) or DA-9082 (100 mg/kg) reduced fasting insulin levels in HFD mice ${ }^{(38,42)}$. Dioscin also significantly reduced fasting insulin levels in $\mathrm{KK}-\mathrm{A}^{\mathrm{y}}$ mice at all three doses 20 , 40 and $80 \mathrm{mg} / \mathrm{kg}^{(35)}$.

Homoeostatic model assessment of insulin resistance, insulin sensitivity index, insulin tolerance test, insulin-glucose ratio and metabolic clearance rate. HOMA-IR was measured in two of the ten studies, while insulin sensitivity index was measured in one, ITT was measured in two, insulin-glucose ratio was measured in one and MCR was measured in one (Table 3$)^{(35,36,38,42,43)}$. Administration of the dioscin in HFD mice and $\mathrm{KK}-\mathrm{A}^{\mathrm{y}}$ significantly reduced HOMA-IR, insulin sensitivity index and glucose following an ITT at all three doses $(20-80 \mathrm{mg} / \mathrm{kg})^{(35)}$, HOMA-IR in ref. [38] reduced in 10, $20 \mathrm{mg} / \mathrm{kg}$ dioscin groups, while administration of diosgenin in OLEFT mice significantly increased MCR and DA-9802 in reduced insulin-glucose ratio significantly ${ }^{(42,43)}$. ITT in ref. [36] was significant in CYP $20 \mathrm{mg} / \mathrm{kg}$. 
NS British Journal of Nutrition

Table 2. Key characteristics of the selected studies

\begin{tabular}{|c|c|c|c|c|c|c|c|c|c|c|}
\hline \multirow[b]{2}{*}{ Article } & \multirow{2}{*}{$\begin{array}{l}\text { Country } \\
\text { of origin }\end{array}$} & \multirow{2}{*}{$\begin{array}{l}\text { Study } \\
\text { design }\end{array}$} & \multicolumn{2}{|c|}{ Animal model } & \multirow[b]{2}{*}{ Yam species } & \multirow{2}{*}{$\begin{array}{l}\text { Yam/its } \\
\text { extract }\end{array}$} & \multirow{2}{*}{$\begin{array}{l}\text { Mode of } \\
\text { administration }\end{array}$} & \multirow[b]{2}{*}{ Study duration } & \multirow{2}{*}{$\begin{array}{l}\text { Biological } \\
\text { parameter }\end{array}$} & \multirow[b]{2}{*}{ Extraction method } \\
\hline & & & Diabetic & Control & & & & & & \\
\hline Xu et al., 2020 & China & $\mathrm{RCT}$ & HFD KK-Ay mice & HFD KK-Ay mice & $\begin{array}{l}\text { D. nipponica } \\
\text { rhizomes }\end{array}$ & Dioscin & $\begin{array}{l}\text { Gavage - OD/8 } \\
\text { weeks }\end{array}$ & 8 weeks & $\begin{array}{l}\text { - FBG, OGTT, } \\
\text { HbA, } \\
\text { - IL, HOMA-IR, } \\
\text { ISI, ITT } \\
\text { - weight } \\
\text { - TC, TAG, LDL, } \\
\text { HDL, NEFA }\end{array}$ & $-50-60 \%$ aqueous ethanol \\
\hline Cheng et al., 2019 & China & $\mathrm{RCT}$ & $\begin{array}{l}\text { HFD C57BL/6 } \\
\text { mice }\end{array}$ & $\begin{array}{l}\text { HFD C57BL/6 } \\
\text { mice }\end{array}$ & Chinese yam & CYP & Orally - OD/8 weeks & 14 weeks & $\begin{array}{l}\text { - FBG } \\
\text { - ITT } \\
\text { - Weight } \\
\text { - TC, TAG, LDL, } \\
\quad \text { HDL }\end{array}$ & $\begin{array}{l}\text { - Alcohol extraction using water bath } \\
\text { - } \mathrm{DdH}_{2} \mathrm{O} \text { used for washing }\end{array}$ \\
\hline Li et al., 2019 & China & $\mathrm{RCT}$ & $\begin{array}{l}\text { HFD C57BL/6 } \\
\text { mice }\end{array}$ & $\begin{array}{l}\text { HFD C57BL/6 } \\
\text { mice }\end{array}$ & & Dioscin & $\begin{array}{l}\text { Gavage - for } 12 \\
\text { weeks }\end{array}$ & 12 weeks & $\begin{array}{l}\text { - FBG } \\
\text { - IL, HOMA-IR, } \\
\text { Adipo-IRI } \\
\text { - weight } \\
\text { - TC, TAG, } \\
\text { NEFA } \\
\text { - TF, adiposity } \\
\text { index }\end{array}$ & - Not reported \\
\hline Wu et al., 2018 & Taiwan & $\mathrm{RCT}$ & $\begin{array}{l}\text { HFD C57BL/6 } \\
\text { mice }\end{array}$ & $\begin{array}{l}\text { HFD C57BL/6 } \\
\text { mice }\end{array}$ & D. alata & Dioscorin & $\begin{array}{l}\text { Gavage - OD/19 } \\
\text { weeks }\end{array}$ & 19 weeks & $\begin{array}{l}\text { - OGTT } \\
\text { - Weight } \\
\text { - TC, TAG, LDL }\end{array}$ & $\begin{array}{l}\text { - Homogenised with Tris buffer } \\
\text { - Eluted with } \mathrm{NaCl} \text { and dialysed with } \\
\text { deionised water }\end{array}$ \\
\hline $\begin{array}{l}\text { Hashidume et al., } \\
\text { 2018A }\end{array}$ & Japan & $\mathrm{RCT}$ & HFD KK-A ${ }^{y}$ mice & HFD KK-Ay mice & D. batatas & $\begin{array}{l}\text { Sanyaku } \\
\text { Diosgenin }\end{array}$ & Orally - for 11 weeks & 11 weeks & $\begin{array}{l}\text { - FBG } \\
\text { - Weight } \\
\text { - TAG } \\
\text { - WAT }\end{array}$ & $\begin{array}{l}\text { - Diosgenin purchased from Sigma } \\
\text { Chemical } \\
\text { - Sanyaku purchased in freeze-dried } \\
\text { powder form }\end{array}$ \\
\hline Sato et al., 2017 & Japan & $\mathrm{RCT}$ & OLEFT mice & LETO mice & D. esculenta & Diosgenin & $\begin{array}{l}\text { Added to diet with } \\
\text { ad libitum access }\end{array}$ & 8 weeks & $\begin{array}{l}\text { - FBG } \\
\text { - MCR } \\
\text { - Weight }\end{array}$ & - Not reported \\
\hline Shih et al., 2015 & Taiwan & $\mathrm{RCT}$ & HFD MW rats & HFD MW rats & & Dioscorin & Orally - OD/5 weeks & 10 weeks & $\begin{array}{l}\text { - OGTT } \\
\text { - Weight } \\
\text { - SBP/DBP }\end{array}$ & - Not reported \\
\hline Moon et al., 2014 & Korea & $\mathrm{RCT}$ & $\begin{array}{l}\text { HFD } d b / d b \text { mice } \\
\text { (C57BLKS/J) }\end{array}$ & $\begin{array}{l}\text { HFD } d b / d b \text { mice } \\
\text { (C57BLKS/J) }\end{array}$ & $\begin{array}{l}\text { D. japonica \& } \\
\text { D. nipponica }\end{array}$ & DA-9081 & $\begin{array}{l}\text { Orally }-\mathrm{OD} / 12 \\
\text { weeks }\end{array}$ & 12 weeks & $\begin{array}{l}\text { - FBG } \\
\text { - Weight }\end{array}$ & $\begin{array}{l}\text { - } 50 \% \text { aqueous ethanol } \\
\text { - At room temperature for } 48 \mathrm{~h} \\
\text { - Filtered and concentrated }\end{array}$ \\
\hline Kim et al., 2012 & Korea & RCT & $\begin{array}{l}\text { HFD C57BL/6 } \\
\text { mice }\end{array}$ & $\begin{array}{l}\text { HFD C57BL/6 } \\
\text { mice }\end{array}$ & D. batatas & DA-9082 & $\begin{array}{l}\text { Gavage - OD/7 } \\
\text { weeks }\end{array}$ & 12 weeks & $\begin{array}{l}\text { - FBG, OGTT } \\
\text { - IL, IGR } \\
\text { - Weight }\end{array}$ & $\begin{array}{l}\text { - Water-ethanol solution for } 24 \mathrm{~h} \\
\text { - Filtered and concentrated }\end{array}$ \\
\hline Hsu et al., 2007 & Taiwan & RCT & $\begin{array}{l}\text { High fructose } \\
\text { diet MW rats }\end{array}$ & MW rats & $\begin{array}{l}\text { D. opposite } \\
\text { thumb }\end{array}$ & $\begin{array}{l}\text { D. opposite } \\
\text { thumb }\end{array}$ & Orally through water & 4 weeks & $\begin{array}{l}- \text { FBG, IPTT, } \\
\mathrm{HbA}_{1} \mathrm{C}\end{array}$ & $\begin{array}{l}\text { - Purchased from Shen Chang } \\
\text { Pharmaceutical Co., Ltd }\end{array}$ \\
\hline
\end{tabular}

RCT, randomised control trial; HFD, high fat diet; MW, male Wister; D., Dioscorea; CYP, Chinese yam polysaccharides; OD, once daily; FBG, fasting blood glucose; OGTT, oral glucose tolerance test; HbA1C, glycated haemoglobin; IPTT, intraperitoneal glucose tolerance test; IL, insulin level; HOMA-IR, homoeostatic model assessment for insulin resistance; ISI, insulin sensitivity index; ITT, insulin tolerance test; IGR, insulin-glucose ratio; MCR, metabolic clearance rate; AdipoIRI, adiposity insulin resistance test; TC, total cholesterol; TF, total fat; WAT, white adiposity tissue; SBP, systolic blood pressure; DBP, diastolic blood pressure; DdH ${ }_{2} \mathrm{O}$, double distilled water. 
Table 3. Effects of yam consumption on glycaemic parameters measured in the selected studies

\begin{tabular}{|c|c|c|c|c|c|c|c|c|c|c|c|c|c|}
\hline \multirow[b]{2}{*}{ Article } & \multirow[b]{2}{*}{ Yam/extract } & \multirow[b]{2}{*}{ Model } & \multirow[b]{2}{*}{ Dose } & \multicolumn{2}{|c|}{ Glucose } & \multirow[b]{2}{*}{$\mathrm{HbA}_{1} \mathrm{C}(\%)$} & \multirow[b]{2}{*}{ Insulin } & \multirow[b]{2}{*}{ IGR } & \multirow[b]{2}{*}{ HOMA-IR } & \multirow[b]{2}{*}{ ISI } & \multirow[b]{2}{*}{ MCR } & \multirow[b]{2}{*}{ ITT } & \multirow[b]{2}{*}{ Adipo-IRI } \\
\hline & & & & FBG & GTT & & & & & & & & \\
\hline \multirow[t]{3}{*}{ Xu et al., 2020} & Dioscin & HFD KK-A ${ }^{y}$ mice & $20 \mathrm{mg} / \mathrm{kg}$ & NS & $0-120 \min \downarrow^{\star *}$ & $\downarrow^{\star *}$ & $\downarrow^{\star *}$ & & $\downarrow^{\star *}$ & $\downarrow^{\star *}$ & & $\downarrow^{\star *}$ & \\
\hline & & & $40 \mathrm{mg} / \mathrm{kg}$ & $\downarrow^{\star \star}$ & & $\downarrow^{\star *}$ & $\downarrow^{\star *}$ & & $\downarrow^{\star \star}$ & $\downarrow^{\star *}$ & & $\downarrow^{\star *}$ & \\
\hline & & & $80 \mathrm{mg} / \mathrm{kg}$ & $\downarrow^{\star \star}$ & & $\downarrow^{\star *}$ & $\downarrow^{\star *}$ & & $\downarrow^{\star *}$ & $\downarrow^{\star *}$ & & $\downarrow^{\star *}$ & \\
\hline \multirow{3}{*}{ Li et al., 2019} & & HFD mice & $5 \mathrm{mg} / \mathrm{kg}$ & NS & & & NS & & NS & & & & $\downarrow^{*}$ \\
\hline & & & $10 \mathrm{mg} / \mathrm{kg}$ & $\downarrow^{*}$ & & & $\downarrow^{*}$ & & $\downarrow^{*}$ & & & & $\downarrow^{* *}$ \\
\hline & & & $20 \mathrm{mg} / \mathrm{kg}$ & $\downarrow^{*}$ & & & $\downarrow^{\star *}$ & & $\downarrow^{*}$ & & & & $\downarrow^{\star *}$ \\
\hline \multirow[t]{4}{*}{ Wu et al., 2018} & Dioscorin & HFD mice & $80 \mathrm{mg} / \mathrm{kg}$ & & $30 \min \downarrow^{\star *}$ & & & & & & & & \\
\hline & & & & & $60 \min \downarrow^{\star *}$ & & & & & & & & \\
\hline & & & & & $90 \min \downarrow^{* *}$ & & & & & & & & \\
\hline & & & & & $120 \min \downarrow^{*}$ & & & & & & & & \\
\hline Shih et al., 2015 & & HFD rats & $50 \mathrm{mg} / \mathrm{kg}$ & & $0-120 \min \downarrow^{*}$ & & & & & & & & \\
\hline \multirow[t]{2}{*}{ Moon et al., 2014} & DA-9801 & $d b / d b$ mice & $30 \mathrm{mg} / \mathrm{kg}$ & $\downarrow^{*}$ & & & & & & & & & \\
\hline & & & $100 \mathrm{mg} / \mathrm{kg}$ & $\downarrow^{*}$ & & & & & & & & & \\
\hline Kim et al., 2012 & DA-9802 & HFD mice & $100 \mathrm{mg} / \mathrm{kg}$ & $\downarrow^{*}$ & $\begin{array}{l}0-5 \mathrm{~min}: \mathrm{NS} \\
30-120 \mathrm{~min} \downarrow^{*}\end{array}$ & & $\downarrow^{*}$ & $\downarrow^{*}$ & & & & & \\
\hline \multirow[t]{3}{*}{ Hsu et al., 2007} & D. Opposita Thunb. & FD rats & DHW & $\downarrow^{*}$ & $\downarrow^{*}$ & $\downarrow^{*}$ & & & & & & & \\
\hline & & & DHW/D. & NS & NS & NS & & & & & & & \\
\hline & & & D. $4.2 \mathrm{mg} / \mathrm{kg}$ & $\downarrow^{*}$ & $\downarrow^{*}$ & $\downarrow^{*}$ & & & & & & & \\
\hline \multirow[t]{2}{*}{ Cheng et al., 2019} & CYP & HFD mice & $10 \mathrm{mg} / \mathrm{kg}$ & NS & & & & & & & & NS & \\
\hline & & & $20 \mathrm{mg} / \mathrm{kg}$ & $\downarrow^{*}$ & & & & & & & & $\downarrow^{*}$ & \\
\hline Sato et al., 2017 & Diosgenin & $\begin{array}{l}\text { ND OLEFT } \\
\text { mice }\end{array}$ & $0.3 \%$ added to ND & $\begin{array}{l}6 \text { weeks } \downarrow^{*} \\
8 \text { weeks } \downarrow^{*}\end{array}$ & & & & & & & $\uparrow^{*}$ & & \\
\hline Hashidume et al., 2018 & Sanyaku Diosgenin & HFD KK-A $\mathrm{A}^{\mathrm{y}}$ mice & $0.05 \%$ added to HFD & $\begin{array}{l}\downarrow^{*} \\
\text { NS }\end{array}$ & & & & & & & & & \\
\hline
\end{tabular}

NS, not significant; HFD, high fat diet; FD, fructose diet; ND, normal diet; FBG, fasting blood glucose; GTT, glucose tolerance test; IGR, insulin-glucose ratio; ISI, insulin sensitivity index; MCR, metabolic clearance rate; ITT, insulin tolerance test; Adipo-IRI, adiposity insulin resistance index.

Arrows indicate direction of change;

${ }^{*} P<0.05 ;{ }^{* *} P<0.01$. 
Table 4. Effects of yam consumption on parameters associated with T2DM measured in the selected studies

\begin{tabular}{|c|c|c|c|c|c|c|c|c|c|c|c|c|c|c|c|}
\hline \multirow[b]{2}{*}{ Article } & \multirow[b]{2}{*}{ Yam/extract } & \multirow[b]{2}{*}{ Model } & \multirow[b]{2}{*}{ Dose } & \multirow[b]{2}{*}{$\Delta \mathrm{Wt}}$. & \multicolumn{5}{|c|}{ Lipid profile } & \multirow[b]{2}{*}{$\mathrm{BP}$} & \multicolumn{5}{|c|}{ Inflammation } \\
\hline & & & & & TAG & TC & LDL & NEFA & $\mathrm{HDL}$ & & Lpn & IL $1 \beta$ & IL10 & MMP & $\mathrm{NF}-\mathrm{\kappa B}$ \\
\hline \multirow[t]{3}{*}{ Xu et al., 2020} & Dioscin & HFD KK-A $\mathrm{A}^{\mathrm{y}}$ mice & $20 \mathrm{mg} / \mathrm{kg}$ & $\downarrow^{*}$ & $\downarrow^{* *}$ & $\downarrow^{* *}$ & $\downarrow^{* *}$ & $\downarrow^{*}$ & $\uparrow^{*}$ & & & & & & \\
\hline & & & $40 \mathrm{mg} / \mathrm{kg}$ & $\downarrow^{*}$ & $\downarrow^{\star *}$ & $\downarrow^{* *}$ & $\downarrow^{\star *}$ & $\downarrow^{\star \star}$ & $\uparrow^{*}$ & & & & & & \\
\hline & & & $80 \mathrm{mg} / \mathrm{kg}$ & $\downarrow^{*}$ & $\downarrow^{\star *}$ & $\downarrow^{\star *}$ & $\downarrow^{\star *}$ & $\downarrow^{\star \star}$ & $\uparrow^{*}$ & & & & & & \\
\hline \multirow[t]{3}{*}{ Li et al., 2019} & & HFD mice & $5 \mathrm{mg} / \mathrm{kg}$ & NS & NS & NS & & NS & & & & & & & \\
\hline & & & $10 \mathrm{mg} / \mathrm{kg}$ & $\downarrow^{*}$ & $\downarrow^{*}$ & $\downarrow^{*}$ & & NS & & & & & & & \\
\hline & & & $20 \mathrm{mg} / \mathrm{kg}$ & $\downarrow^{*}$ & $\downarrow^{*}$ & $\downarrow^{*}$ & & $\downarrow^{*}$ & & & & & & & \\
\hline Wu et al., 2018 & Dioscorin & HFD mice & $80 \mathrm{mg} / \mathrm{kg}$ & $\downarrow^{*}$ & NS & $\downarrow^{*}$ & $\downarrow^{*}$ & & & & & & & & \\
\hline Shih et al., 2015 & & HFD rats & $50 \mathrm{mg} / \mathrm{kg}$ & NS & & & & & & $\begin{array}{l}\text { SBP } \downarrow^{*} \\
\text { DBP NS }\end{array}$ & & & & & \\
\hline Moon et al., 2014 & DA-9801 & $d b / d b$ mice & $\begin{array}{l}30 \mathrm{mg} / \mathrm{kg} \\
100 \mathrm{mg} / \mathrm{kg}\end{array}$ & $\begin{array}{l}\text { NS } \\
\text { NS }\end{array}$ & & & & & & & & & & & \\
\hline Kim et al., 2012 & DA-9802 & HFD mice & $100 \mathrm{mg} / \mathrm{kg}$ & NS & & & & & & & & & & & \\
\hline Hsu et al., 2007 & D. Opposita Thunb. & FD rats & $\begin{array}{l}\text { DHW } \\
\text { DHW/D. } \\
\text { D. } 4.2 \mathrm{mg} / \mathrm{kg}\end{array}$ & & & & & & & & & & & & \\
\hline \multirow[t]{2}{*}{ Cheng et al., 2019} & CYP & HFD mice & $10 \mathrm{mg} / \mathrm{kg}$ & NS & NS & NS & NS & & $\uparrow^{*}$ & & NS & $\downarrow^{*}$ & $\downarrow^{*}$ & $\downarrow^{*}$ & $\downarrow^{*}$ \\
\hline & & & $20 \mathrm{mg} / \mathrm{kg}$ & $\downarrow^{*}$ & NS & $\downarrow^{*}$ & $\downarrow^{*}$ & & $\uparrow^{*}$ & & $\downarrow^{*}$ & $\downarrow^{* *}$ & $\downarrow^{* *}$ & $\downarrow^{* *}$ & $\downarrow^{* *}$ \\
\hline Sato et al., 2017 & Diosgenin & $\begin{array}{l}\text { ND OLEFT } \\
\text { mice }\end{array}$ & $0.3 \%$ added to ND & NS & & & & & & & & & & & \\
\hline Hashidume et al., 2018 & Sanyaku Diosgenin & HFD KK-Ay mice & $0.05 \%$ added to HFD & NS & NS & & & & & & & & & & \\
\hline
\end{tabular}

NS, not significant; HFD, high fat diet; FD, fructose diet; ND, normal diet; $\triangle \mathrm{WT}$, change in body weight; TC, total cholesterol; BP, blood pressure; SBP, systolic blood pressure; DBP, diastolic blood pressure; Lpn, leptin; MMP, matrix metalloproteinases; NF-kB, nuclear factor kappa-light-chain-enhancer of activated $B$ cells.

Arrows indicate direction of change;

${ }^{*} P<0.05 ;{ }^{\star *} P<0.01$ 
Adiposity insulin resistance indexOne study measured adiposity insulin resistance index and showed that dioscin treatment $(5-10 \mathrm{mg} / \mathrm{kg})$ resulted in significant reduction $(P<0 \cdot 05)^{(38)}$.

\section{Other factors related to glycaemic control}

Body weight changes, total fats, white adiposity tissue and adiposity index. Nine of the ten studies measured body weight; of these, four observed a significant decrease in body weight regardless of diabetic model (HFD, KK- $\mathrm{A}^{\mathrm{y}}$ or $\mathrm{C} 57 \mathrm{BL} / 6$ ) or yam extract (dioscin, CYP, dioscorin; $P<0 \cdot 05)^{(35,36,38,40)}$. Li et al. found that the decrease in body weight following dioscin treatment $(10-20 \mathrm{mg} / \mathrm{kg})$ was due to a $14 \%$ decrease in total fat $(P<0.05)$. However, ref. [33,39,41-43] did not observe any changes in body weight nor changes in white adiposity tissue following treatment with sanyaku, diosgenin, dioscorin, DA9801 and DA-9802 (Table 4).

Lipid profile (total cholesterol, TAG, LDL, HDL and NEFA). Five studies measured the lipid profile biomarkers ${ }^{(35,36,38,40,41)}$, these included TC, TAG, LDL, HDL and NEFA (Table 4); of these, four found changes in some lipid biomarkers, only ref. [41] observed no differences between treatment and control. References [35] and [38] revealed significant reductions in TC, TAG, LDL and NEFA following dioscin and treatment $(P<0.05)$ in both KK-A $\mathrm{A}^{\mathrm{y}}$ and HFD diet mice. These results were partially supported by ref. [36,40]; while they observed significant reduction in TC and LDL following dioscorin and CYP treatment, respectively, in HFD model, no changes in TAG were observed. In addition, both refs [35,36] found that dioscin and CYP also increased HDL levels.

Blood pressure. SBP and DBP were measured in only one of ten studies. Reference [39] showed a significant decrease in SBP $(P<0 \cdot 05)$, but not DBP (Table 4).

Inflammatory markers. One of the ten studies measured markers of inflammation and adipocytokines, these included leptin, IL1 $\beta$, IL-10, MMP and NF-kB. Cheng et al. showed that administration of CYP induced a significant decrease in all the markers suggesting an anti-inflammatory effect of CYP (Table 4).

\section{Discussion}

The number of people suffering from T2DM is increasing worldwide and has become a global public health problem. New treatment strategies are increasingly needed, and many studies have indicated that natural food constituents, such as resistance starch and bioactive compounds (e.g. phytochemicals), could be incorporated into a healthy balanced diet to aid in the prevention or management of T2DM.

Yam (Dioscorea spp.) is the fourth most important tuber crop after potatoes, cassava and sweet potatoes and contains a good source of essential dietary supplements such as protein, well-balanced essential amino acids and many dietary minerals ${ }^{(44,45)}$. Recently, interest has focused on yam as a potential insulin mimetic; thus, we searched the current literature to investigate whether yam and/or its extracts have the potential to help manage T2DM. We observed that the consumption of yam and/ or its extracts had a beneficial effect on numerous glycaemic parameters including FBG, insulin, Hb1Ac and HOMA-IR. Additionally the consumption of yam and/or its extracts helped improvements in adiposity and circulating lipids, which are known to influence the development of T2DM.

However much of the work conducted on the effects of yam on T2DM has been shown in animal models and therefore further research is required in human participants, although ref. [46] has shown that the consumption of brown yam flour improved glycaemia in healthy subjects compared with other yam flours. In this review, we focused on studies conducted in models in which T2DM was induced by diet or in animals genetically predisposed to developing T2DM rather than those in which diabetes was induced by an injection of STZ, which is a model of type 1 diabetes. High fat feeding in mice leads to obesity, hyperinsulinaemia and altered glucose homoeostasis due to insufficient compensation by islets, thus modelling the human situation more accurately ${ }^{(47)}$, while STZ administration damages pancreatic $\beta$-cells depicting type 1 diabetes $^{(48)}$. However, we also included rodent models in which T2DM develops spontaneously this included the OLEFT model, in which animals inherit diabetes, KK- $\mathrm{A}^{\mathrm{y}}$ mice that develop obesity and severe hyperinsulinaemia ${ }^{(49)}$, again mimic human predisposition to diabetes. Furthermore, in these models, insulin sensitivity can be reversed via dietary manipulation and/or pharmacological administration as well as enable us to understand possible mechanisms ${ }^{(49)}$.

All papers identified in this review showed that the consumption of yam and/or its extracts at various doses improved glycaemia by improving fasting glucose levels and insulin sensitivity. As mentioned earlier, starch is the most abundant component of yam; cooking alters the properties of the starch making it more resistant to digestion. Resistant starch has been shown to prevent hyperglycaemia and reduce the risk of diabetes ${ }^{(12,50,51)}$ and lower serum TAG and LDL-cholesterol due to reduction in fat absorption $^{(52,53)}$. However, all studies identified in this review used extracts to treat the rodents; thus, it is unlikely that an increase in resistance starch was responsible for the observed effects, but does highlight that the consumption of yam or its extracts has similar effects on glycaemia.

Another possible reason for the observed improvements in glycaemia maybe due to the inhibition of $\alpha$-glucosidase; indeed yam and its extracts have been shown to be potent inhibitors of this enzyme ${ }^{(27,54)} \cdot \alpha$-glucosidase is located on the brush border of the small intestine and breaks down starch to glucose, and many $\alpha$-glucosidase inhibitors, such as quercetin and acarbose, have been developed into clinical drugs to reduce blood glucose levels ${ }^{(55-58)}$. Not only do these inhibitors reduce FBG $^{(59)}$ they also reduce post-prandial hyperglycaemia, thus reducing $\mathrm{Hb} 1 \mathrm{Ac}^{(60)}$. However, only two of the eight studies measured this, but both found significant decreases ${ }^{(35,37)}$. These inhibitors can also influence the release of the incretin glucagon-like peptide 1 , in support ref. [23] showed that allantoin (a yam extract) can increase glucagon-like peptide 1 release in a rat model of STZ induced diabetes. Indeed, we found that yam and/or its extracts treatment led to a decrease in plasma insulin in the three studies, which measured insulin levels ${ }^{(35,38,42)}$. Furthermore, these inhibitors can reduce lipid deposition and reduce adipocyte size and 
TAG and $\mathrm{LDL}^{(23,57,58)}$; indeed, we observed reductions in TAG, LDL, NEFA and TC in five of the ten studies ${ }^{(35,36,38,40,41)}$. Thus, further supporting the notion that the consumption of yam and/or its extracts results in the inhibition of $\alpha$-glucosidase to exert its effects. However, the potency of inhibition of $\alpha$-glucosidase by yam and/or its extracts is dependent on the solvent used for extraction and may be one of the reasons why there was difference in the lowest working dose observed in the studies, even when the same yam extract was utilised ${ }^{(27,54)}$. Indeed, ref. [35] used sodium carboxymethyl cellulose, while ref. [38] used saline to dissolve dioscin.

Other modes of action that yam and/or its extracts could improve glycaemic parameters are via the amelioration of oxidative and inflammatory responses. The consumption of HFD and high lipid profile levels can lead to the development of oxidative stress and systematic inflammation ${ }^{(61)}$, which results in decreased insulin sensitivity leading to hyperinsulinaemia and hyperglycaemia causing a pre-diabetic state. If uncontrolled, this can hinder the ability of the $\beta$-cells to meet demand leading to the development of diabetes ${ }^{(62)}$. This further exacerbates oxidative stress and inflammation leading to complications such as hypertension ${ }^{(63)}$. Numerous studies support the notion that yam and/or its extracts have antioxidant and anti-inflammatory properties ${ }^{(64-66)}$.

Indeed, we found that treatment with CYP was found to decrease pro-inflammatory markers NF- $\mathrm{BB}, \mathrm{MMP}-3$, IL-1B and anti-inflammatory marker IL-10 ${ }^{(36)}$. IL-10 increases with obesity to protect against the disruption of insulin signalling; thus, ref. [36] concluded that CYP acted to reduce pro-inflammatory cytokines rather than stimulating anti-inflammatory cytokines. Extracts from $D$. batatas decrease the expression of pro-inflammatory cytokines TNF- $\alpha$, monocyte chemoattractant protein-1 (MCP-1) and IL-6 in obese rodents $^{(67)}$. In addition, the levels of PPAR $\gamma$ coactivator $1 \alpha$ (PGC- $1 \alpha$ ) in the pancreas return to basal expression levels in those animals of normal weight, similar to the effects of the drug metfor$\min ^{(68)}$. PGC- $1 \alpha$ deficiency in the pancreas leads to an increase in pro-inflammatory markers production via $\mathrm{NF}-\kappa \mathrm{B}$, which in turn can lead to further damage of the pancreatic tissue ${ }^{(69)}$.

In support of the antioxidant properties, we found a decrease in Hb1Ac in two of the eight studies ${ }^{(35,70)}$. Hb1Ac is a known marker associated with increased oxidative stress ${ }^{(71)}$. In addition, we observed in one study that treatment with dioscorin resulted in a decrease in SBP possibly via angiotensin converting enzyme and vasorelaxation ${ }^{(39,70)}$. Many studies have implicated oxidative stress in hypertension, as reactive oxygen species influence vascular, renal and cardiac function and structure ${ }^{(72)}$. Further evidence of antioxidant/anti-inflammatory properties arises for the fact that yam and/or its extracts can restore the activity of the phosphoinositide 3 kinase/protein kinase B (Akt) and PPAR $\gamma$ pathways, both known to be suppressed in diseases associated with oxidative stress and inflammation ${ }^{(38)}$. Additionally, ref. [73] reported that Sanggua Drink extract, which consists of Dioscorea, might alleviate insulin resistance in HFD fed rodents via the induction of the PI3K/Akt signalling pathway.

The antioxidant/inflammatory properties of yam and/or its extracts may be due to the high phenolic and flavonoid content; these phenolic and flavonoids can reduce reactive oxygen species and reactive nitrogen species protecting pancreatic $\beta$-cells disruption $^{(74,75)}$. Indeed, dioscorin has both dehydroascorbate reductase and monodehydroascorbate reductase activity enabling the generation of ascorbate which in turn to reduce the levels of reactive oxygen species ${ }^{(76)}$, thus proposed to be a good reducing agent. Furthermore, in STZ treated mice both allantoin and diosgenin can increase superoxide dismutase activity and the levels of reduced glutathione suggesting a reduction in oxidative stress. Moreover, allantoin treatment in STZ rats has been shown to reduce $\beta$-cell granulation suggesting that yam may play a potential role in protecting $\beta$-cells function and preventing granulation.

Many of the studies focused on the effects of yam and/or its extract on diabetes have investigated the indirect mechanism of improving glycaemia as discussed above. However, evidence for whether yam and/or its extracts have direct effect on the pancreatic $\beta$-cells or other cell types is limited, despite some evidence of a possible direct mechanism. Pancreatic lipase is released from the pancreas causing a reduction in the absorption and digestion of dietary TAG. D. opposite has been shown to inhibit pancreatic lipase secretion to a similar level to orlistat (the only FDA approved drug that inhibits pancreatic lipase to prevent $30 \%$ for fat absorption $)^{(77-79)}$. This could be associated with changes in lipid profile observed in five of the studies ${ }^{(35,36,38,40,41)}$ and decrease in body weight observed by four of the studies ${ }^{(35,36,38,40)}$. Further evidence for direct effects on the pancreas is the ability of yam, particularly D. batatas and allantoin, to prevent the loss of pancreatic mass by protecting against loss of islets, structural damage and atrophy in STZHFD mice ${ }^{(68,80)}$; however, this effect could potentially be indirect via amelioration of inflammation and oxidative stress, thus warrants further studies. There is also evidence that yam and/or its extracts improve glycaemia via improvements in adipose and muscle tissue. Dioscin and D. batatas administration decreases visceral adipose tissues and lipid profile, although this is thought to be via the improvements in inflammation. Interestingly, there is evidence of direct effects on muscle, administration of allantoin improved glucose uptake in skeletal muscle isolated from STZ-diabetic rats, possibly via increased translocation of GLUT 4, leading to increased MCR as suggested by ref. [21,43]. Furthermore, administration of D. batatas or allantoin in STZdiabetic rodents increased microfibre number and area. It is well known that insulin resistance is manifested by a decrease in insulin stimulate glucose uptake in skeletal muscle. Nonetheless, the mechanism by which yam and/or extracts influence this is unknown and warrants further investigation.

\section{Limitation}

While all the studies identified in this review and others conducted in STZ rodents agree that the consumption of the various yam and/or its extracts improves diabetic outcomes, there are some limitations to the study. These include not being able to agree on one species of yam or extract, or specific dose or length of consumption. Furthermore, the data extracted from the studies were descriptive and not actual values; therefore, a metaanalysis was not possible. However, the biggest limitation of this study is the lack of human studies. These are required to determine whether the effects observed in animal studies can be 
translated and, moreover, allow us to assess whether the consumption of yam mimics those observed following the consumption of the extracts, to assess whether the process of cooking yam alters glycaemia and finally to understand whether lifestyle and habitual diet could influence the effects observed.

\section{Conclusion}

In summary, yam and its extracts have the potential to act as functional foods in the treatment of T2DM in numerous ways. However, further studies are required to understand potential mechanisms particularly in understanding the molecular pathways associated with insulin and glucose in the various important tissues (pancreas, muscle, adipose tissue) and to understand whether these are direct or indirect. Furthermore, human studies are required alongside studies comparing yam to similar to reliably inform dietetic practice, guidelines and policy makers.

\section{Acknowledgements}

This systematic review was funded by a scholarship award to W. Z. A. by the Saudi Arabian Government and the Albaha University, Saudi Arabia. The funders were not involved in the study design, collection, analysis and interpretation of the data, nor in the writing of the paper. W. Z. A., A. M., P. R. and P. H. J. designed the research and wrote the paper. W. Z. A. and A. M. conducted the research. All authors have read and approved the final manuscript. P. H. J. had primary responsibility for the final manuscript.

The authors declare that they have no conflicts of interest.

\section{Supplementary material}

For supplementary material referred to in this article, please visit https://doi.org/10.1017/S0007114521003706

\section{References}

1. Saeedi P, Petersohn I, Salpea P, et al. (2019) Global and regional diabetes prevalence estimates for 2019 and projections for 2030 and 2045: results from the International Diabetes Federation Diabetes Atlas. Diabetes Res Clin Pract 157, 107843.

2. International Diabetes Federation (IDF) (2019) Facts \& Figures. https://www.idf.org/aboutdiabetes/what-is-diabetes/factsfigures.html (accessed October 2020).

3. Wild S, Roglic G, Green A, et al. (2004) Global prevalence of diabetes: estimates for the year 2000 and projections for 2030. Diabetes Care 27, 1047-1053.

4. Colledge NR, Walker BR \& Ralston SH (2010) Davidson's Principles and Practice of Medicine, 21st ed. London: Churchill Livingstone Elsevier.

5. Erion DM, Park HJ \& Lee HY (2016) The role of lipids in the pathogenesis and treatment of type 2 diabetes and associated co-morbidities. BMB Rep 49, 139.

6. Dhananjayan R, Koundinya KS, Malati T, et al. (2016) Endothelial dysfunction in type 2 diabetes mellitus. Indian $J$ Clin Biochem 31, 372-379.

7. Constantino MI, Molyneaux L, Limacher-Gisler F, et al. (2013) Long-term complications and mortality in young-onset diabetes: type 2 diabetes is more hazardous and lethal than type 1 diabetes. Diabetes Care 36, 3863-3869.

8. Haak T, Hanaire H, Ajjan R, et al. (2017) Flash glucose-sensing technology as a replacement for blood glucose monitoring for the management of insulin-treated type 2 diabetes: a multicenter, open-label randomized controlled trial. Diabetes Ther $\mathbf{8}$, 55-73.

9. Giorgino F, Laviola L \& Leonardini A (2005) Pathophysiology of type 2 diabetes: rationale for different oral antidiabetic treatment strategies. Diabetes Res Clin Pract 68, S22-S29.

10. Wu Y, Ding Y, Tanaka Y, et al. (2014) Risk factors contributing to type 2 diabetes and recent advances in the treatment and prevention. Int J Med Sci 11, 1185.

11. Samtiya M, Aluko RE, Dhewa T, et al. (2021) Potential health benefits of plant food-derived bioactive components: an overview. Foods 10, 839.

12. Rinaldo D (2020) Carbohydrate and bioactive compounds composition of starchy tropical fruits and tubers, in relation to pre and postharvest conditions: a review. J Food Sci 85, 249-259.

13. Mirmiran P, Bahadoran Z \& Azizi F (2014) Functional foodsbased diet as a novel dietary approach for management of type 2 diabetes and its complications: a review. World J Diabetes 5, 267.

14. Food and Agriculture Organization of the United Nations (FAO) (2018) FAOSTAT Crops. http://www.fao.org/faostat/en/\#data/ QC/visualize (accessed June 2020).

15. Uloko AE, Musa BM, Ramalan MA, et al. (2018) Prevalence and risk factors for diabetes mellitus in Nigeria: a systematic review and meta-analysis. Diabetes Ther 9, 1307-1316.

16. Gatimu SM, Milimo BW \& San Sebastian M (2016) Prevalence and determinants of diabetes among older adults in Ghana. BMC Public Health 16, 1174.

17. Jia W, Gao W \& Tang L (2003) Antidiabetic herbal drugs officially approved in China. Phytother Res 17, 1127-1134.

18. Maithili V, Dhanabal SP, Mahendran S, et al. (2011) Antidiabetic activity of ethanolic extract of tubers of Dioscorea alata in alloxan induced diabetic rats. Indian J Pharmacol 43, 455

19. Guo C, Ding G, Huang W, et al. (2016) Total saponin of Dioscoreae hypoglaucae rhizoma ameliorates streptozotocin-induced diabetic nephropathy. Drug Design Dev Ther 10, 799 .

20. Yu H, Zheng L, Xu L, et al. (2015) Potent effects of the total saponins from dioscorea nipponica makino against streptozotocin-induced type 2 diabetes mellitus in rats. Phytother Res 29, 228-240.

21. Niu CS, Chen W, Wu HT, et al. (2010) Decrease of plasma glucose by allantoin, an active principle of yam (Dioscorea spp.), in streptozotocin-induced diabetic rats. J Agr Food Chem 58, 12031-12035.

22. McAnuff MA, Harding WW, Omoruyi FO, et al. (2005) Hypoglycemic effects of steroidal sapogenins isolated from Jamaican bitter yam, Dioscorea polygonoides. Food Chem Toxicol 43, 1667-1672.

23. Go HK, Rahman M, Kim GB, et al. (2015) Antidiabetic effects of yam (Dioscorea batatas) and its active constituent, allantoin, in a rat model of streptozotocin-induced diabetes. Nutrients $\mathbf{7}$, 8532-8544.

24. Amitani M, Cheng KC, Asakawa A, et al. (2015) Allantoin ameliorates chemically-induced pancreatic $\beta$-cell damage through activation of the imidazoline $\mathrm{I} 3$ receptors. PeerJ 3, e1105.

25. Estiasih T \& Umaro D (2018) Hypoglycemic effect of crude water soluble polysaccharide extracted from tubers of purple and yellow water yam (Dioscorea alata L.) on alloxan-induced hyperglycemia Wistar rats. Progr Nutr 20, 59-67.

26. Lee HR, Kong SY, Sung SH, et al. (2019) DA-9801 and its saponins, dioscin and protodioscin, protect primary cortical 
neurons from hyperglycemia-induced neurotoxicity. J Funct Foods 54, 231-240.

27. Ghosh S, More P, Derle A, et al. (2014) Diosgenin from Dioscorea bulbifera: novel hit for treatment of type II diabetes mellitus with inhibitory activity against $\alpha$-amylase and $\alpha$-glucosidase. PlOS ONE 9, e106039.

28. Ghosh S, Ahire M, Patil S, et al. (2012) Antidiabetic activity of Gnidia glauca and Dioscorea bulbifera: potent amylase and glucosidase inhibitors. Evid-Based Complement Alternat Med 2012, 929051.

29. Islam MS \& Wilson RD (2012) Experimentally induced rodent models of type 2 diabetes. In Animal Models in Diabetes Research, Methods in Molecular Biology, pp. 161-174 [H-G Joost, H Al-Hasani \& A Schürmann, editors]. Totowa, NJ: Humana Press.

30. Moher D, Shamseer L, Clarke M, et al. (2015) Preferred reporting items for systematic review and meta-analysis protocols (PRISMA-P) 2015 statement. Syst Rev 4, 1.

31. Higgins JP, Altman DG, Gøtzsche PC, et al. (2011) The Cochrane Collaboration's tool for assessing risk of bias in randomised trials. BMJ 343, d5928.

32. Hooijmans CR, Rovers MM, de Vries RB, et al. (2014) SYRCLE's risk of bias tool for animal studies. BMC Med Res Methodol 14, 43 .

33. Moon E, Lee SO, Kang TH, et al. (2014) Dioscorea extract (DA9801) modulates markers of peripheral neuropathy in type 2 diabetic db/db mice. Biomol Therapeut 22, 445.

34. Hirst JA, Howick J, Aronson JK, et al. (2014) The need for randomization in animal trials: an overview of systematic reviews. PLOS ONE 9, e98856.

35. Xu LN, Yin LH, Jin Y, et al. (2020) Effect and possible mechanisms of dioscin on ameliorating metabolic glycolipid metabolic disorder in type-2-diabetes. Phytomedicine 67, 153139.

36. Cheng Z, Hu M, Tao J, et al. (2019) The protective effects of Chinese yam polysaccharide against obesity-induced insulin resistance. J Funct Food 55, 238-247.

37. Hsu JH, Wu YC, Liu IM, et al. (2007) Dioscorea as the principal herb of Die-Huang-Wan, a widely used herbal mixture in China, for improvement of insulin resistance in fructose-rich chow-fed rats. J Ethnopharmacol 112, 577-584.

38. Li H, Yu L \& Zhao C (2019) Dioscin attenuates high-fat dietinduced insulin resistance of adipose tissue through the IRS1/PI3K/Akt signaling pathway. Mol Med Rep 19, 1230-1237.

39. Shih SL, Lin YS, Lin SY, et al. (2015) Effects of yam dioscorin interventions on improvements of the metabolic syndrome in high-fat diet-induced obese rats. Botanical Stud 56, 1-9.

40. Wu GC, Lin SY, Liang HJ, et al. (2018) 135-day interventions of yam dioscorin and the dipeptide Asn-Trp (NW) to reduce weight gains and improve impaired glucose tolerances in high-fat diet-induced c57bl/6 mice. J Agr Food Chem 66, 645-652.

41. Hashidume T, Sasaki K, Hirata J, et al. (2018) Effects of sanyaku and its constituent diosgenin on the fasted and postprandial hypertriacylglycerolemia in high-fat-diet-fed KK-A y mice. J Agr Food Chem 66, 9968-9975.

42. Kim S, Jwa H, Yanagawa Y, et al. (2012) Extract from Dioscorea batatas ameliorates insulin resistance in mice fed a high-fat diet. J Med Food 15, 527-534.

43. Sato K, Fujita S \& Iemitsu M (2017) Dioscorea esculentainduced increase in muscle sex steroid hormones is associated with enhanced insulin sensitivity in a type 2 diabetes rat model. FASEB J 31, 793-801.

44. Padhan B \& Panda D (2020) Potential of neglected and underutilized yams (Dioscorea spp.) for improving nutritional security and health benefits. Front Pharmacol 11, 496.
45. Zhang Z, Gao W, Wang R, et al. (2014) Changes in main nutrients and medicinal composition of Chinese yam (Dioscorea opposita) tubers during storage. J Food Sci Tech 51, 2535-2543.

46. Jimoh AK, Adediran SO, Adebisi SA, et al. (2008) Glycaemic response to different preparations of yam in diabetic and non-diabetic Nigerians. Afr J Diabetes Med 16, 19-22.

47. King AJ (2012) The use of animal models in diabetes research. Br J Pharmacol 166, 877-894.

48. Graham ML, Janecek JL, Kittredge JA, et al. (2011) The streptozotocin-induced diabetic nude mouse model: differences between animals from different sources. Comp Med 61, 356-360.

49. Tomino Y (2012) Lessons from the KK-A mouse, a spontaneous animal model for the treatment of human type 2 diabetic nephropathy. Nephro-Urol Mon 4, 524.

50. Raigond P, Ezekiel R \& Raigond B (2015) Resistant starch in food: a review. J Sci Food Agr 95 , 1968-1978.

51. Birt DF, Boylston T, Hendrich S, et al. (2013) Resistant starch: promise for improving human health. Adv Nutr 4, 587-601.

52. Nishimura N, Tanabe H, Yamamoto T, et al. (2011) Raw Chinese yam (Dioscorea opposita) promotes cecal fermentation and reduces plasma non-HDL cholesterol concentration in rats. J Nutr Sci Vitaminol 57, 340-347.

53. Shujun W, Jinglin Y, Hongyan L, et al. (2008) Characterisation and preliminary lipid-lowering evaluation of starch from Chinese yam. Food Chem 108, 176-181.

54. Zhang L, Bai B, Liu X, et al. (2011) $\alpha$-Glucosidase inhibitors from Chinese yam (Dioscorea opposita Thunb.). Food Chem 126, 203-206.

55. Yang W, Wang Y, Li X, et al. (2015) Purification and structural characterization of Chinese yam polysaccharide and its activities. Carbohydr Polymer 117, 1021-1027.

56. Ma CM, Sato N, Li XY, et al. (2010) Flavan-3-ol contents, antioxidative and $\alpha$-glucosidase inhibitory activities of Cynomorium songaricum. Food Chem 118, 116-119.

57. Kado S, Murakami T, Aoki A, et al. (1998) Effect of acarbose on postprandial lipid metabolism in type 2 diabetes mellitus. Diabetes Res Clin Pract 41, 49-55.

58. Vasselli JR, Haraczkiewicz E, Maggio CA, et al. (1983) Effects of a glucosidase inhibitor (acarbose, BAY g 5421) on the development of obesity and food motivated behavior in Zucker (fafa) rats. Pharmacol Biochem Behav 19, 85-95.

59. Van De Laar FA, Lucassen PL, Akkermans RP, et al. (2005) $\alpha$ Glucosidase inhibitors for patients with type 2 diabetes: results from a Cochrane systematic review and meta-analysis. Diabetes Care 28, 154-163.

60. Padhi S, Nayak AK \& Behera A (2020) Type II diabetes mellitus: a review on recent drug based therapeutics. Biomed Pharmacother 131, 110708.

61. Tan SY, Wong JLM, Sim YJ, et al. (2019) Type 1 and 2 diabetes mellitus: a review on current treatment approach and gene therapy as potential intervention. Diabetes Metab Syndrome: Clin Res Rev 13, 364-372.

62. Manna P \& Jain SK (2015) Obesity, oxidative stress, adipose tissue dysfunction, and the associated health risks: causes and therapeutic strategies. Metab Syndrome Relat Disord 13, 423-444.

63. Ormazabal V, Nair S, Elfeky O, et al. (2018) Association between insulin resistance and the development of cardiovascular disease. Cardiovasc Diabetol 17, 1-14.

64. Chiu CS, Deng JS, Chang HY, et al. (2013) Antioxidant and antiinflammatory properties of Taiwanese yam (Dioscorea japonica Thunb. var. pseudojaponica (Hayata) Yamam.) and its reference compounds. Food Chem 141, 1087-1096. 
65. Jin M, Lu Y, Yang JH, et al. (2011) Anti-inflammatory activity of 6-hydroxy-2, 7-dimethoxy-1, 4-henanthraquinone from tuberous roots of yam (Dioscorea batatas) through inhibition of prostaglandin D 2 and leukotriene C 4 production in mouse bone marrow-derived mast cells. Arch Pharmacal Res 34, 1495-1501.

66. Jin M, Suh SJ, Yang JH, et al. (2010) Anti-inflammatory activity of bark of Dioscorea batatas DECNE through the inhibition of iNOS and COX-2 expressions in RAW264. 7 cells via NF-KB and ERK1/2 inactivation. Food Chem Toxicol 48, 3073-3079.

67. Gil H-W, Lee E-Y, Lee J-H, et al. (2015) Dioscorea batatas extract attenuates high-fat diet-induced obesity in mice by decreasing expression of inflammatory cytokines. Med Sci Monit 21, 489-495.

68. Ma J, Meng X, Liu Y, et al. (2020) Effects of a rhizome aqueous extract of Dioscorea batatas and its bioactive compound, allantoin in high fat diet and streptozotocin-induced diabetic mice and the regulation of liver, pancreas and skeletal muscle dysfunction. J Ethnopharmacol 259, 112926.

69. Rius-Pérez S, Torres-Cuevas I, Monsalve M, et al. (2020) Impairment of PGC-1 alpha up-regulation enhances nitrosative stress in the liver during acute pancreatitis in obese mice. Antioxidant 9, 887 .

70. Hsu FL, Lin YH, Lee MH, et al. (2002) Both dioscorin, the tuber storage protein of yam (Dioscorea alata cv. Tainong No. 1, and its peptic hydrolysates exhibited angiotensin converting enzyme inhibitory activities. J Agr Food Chem 50, 6109-6113.

71. Palem SP \& Abraham P (2015) A study on the level of oxidative stress and inflammatory markers in type 2 diabetes mellitus patients with different treatment modalities. J Clin Diagn Res 9, BC04-BC07.

72. Loperena R \& Harrison DG (2017) Oxidative stress and hypertensive diseases. Med Clin 101, 169-193.
73. Cai Y, Wang Y, Zhi F, et al. (2018) The effect of sanggua drink extract on insulin resistance through the PI3K/AKT signaling pathway. Evid-Based Complement Alternat Med 2018, 9407945.

74. Obidiegwu JE, Lyons JB \& Chilaka CA (2020) The dioscorea genus (yam) - an appraisal of nutritional and therapeutic potentials. Foods 9, 1304.

75. Zraika S, Aston-Mourney K, Laybutt DR, et al. (2006) The influence of genetic background on the induction of oxidative stress and impaired insulin secretion in mouse islets. Diabetologia $\mathbf{4 9}$, $1254-1263$.

76. Hou WC, Chen HJ \& Lin YH (1999) Dioscorins, the major tuber storage proteins of yam (Dioscorea batatas Decne, with dehydroascorbate reductase and monodehydroascorbate reductase activities. Plant Sci 149, 151-156.

77. Kim GN, Shin MR, Shin SH, et al. (2016) Study of antiobesity effect through inhibition of pancreatic lipase activity of Diospyros kaki fruit and Citrus unshiu peel. BioMed Res Int 2016, 1723042

78. Seyedan A, Alshawsh MA, Alshagga MA, et al. (2015) Medicinal plants and their inhibitory activities against pancreatic lipase: a review. Evid-Based Complement Alter Med 2015, 973143.

79. Yang MH, Chin YW, Yoon KD, et al. (2014) Phenolic compounds with pancreatic lipase inhibitory activity from Korean yam (Dioscorea opposita). J Enzym Inhib Med Chem 29, 1-6.

80. de Salgado Rêgo T, da Silva Ash L, Pessoa L, et al. (2014) The intake of Yam (Dioscorea bulbifera Linn) attenuated the hyperglycemia and the bone fragility in female diabetic rats. Nutr bosp 29, 370-375.

81. PRISMA (2015) Preferred Reporting Items for Systematic Reviews and Meta-Analyses (PRISMA). http://www.prismastatement.org/Default.aspx (accessed May 2020). 